\title{
Inclusión Educativa desde un enfoque de derechos
}

\section{Entrevista}

Entrevista realizada a Harold Munster, Profesor Investigador de la Facultad de Ciencias Psicológicas de la Universidad Central del Ecuador, Doctor en Ciencias Pedagógicas.

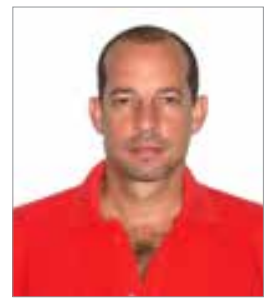

\section{S.A.: ¿Por qué hablar de educación inclusiva, acaso la educación no debería ser inclusiva?}

Harold Munster: Yo no lo veo necesario, más bien, considero que es inadecuado, o sea, no existe educación inclusiva, existen buenas y malas educaciones, existe una educación adecuada cuando hace inclusión educativa; existe una educación inadecuada, insuficiente, cuando no hace inclusión educativa, la condición de inclusiva es inherente como principio de la educación. No importa quién la haga, no importa el posicionamiento, no importa el país, no importa la concepción, al final toda educación, por principio, debe y tiene que ser inclusiva. Cuando se hace inclusión educativa, entonces es una educación que está cumpliendo con su principio de inclusión, está cumpliendo con la garantía de los derechos de grupos de atención prioritaria.

\section{S.A.: ¿Cuándo es inclusiva una educación?}

Harold Munster: Una educación es inclusiva cuando, en principio, se logra garantizar, preservar y defender los derechos de las personas que tienen el servicio de la educación. La inclusión se logra cuando realmente podemos hacer todo un proceso que garantice el acceso adecuado a la educación donde personas en situación de discapacidad, puedan tener garantizados sus derechos a una autonomía funcional, a una participación social y por supuesto, a un desarrollo integral y multifacético. 


\section{S.A.: Pero, se puede pensar que esto no solamente es para las per- sonas que están en situación de discapacidad, ¿qué otro tipo de inclusiones deberíamos precisar?}

Harold Munster: Yo creo que la idea es tomar en consideración todos los grupos de atención prioritarios, y cuando hablo de grupos de atención prioritaria, estoy hablando de aquellos grupos que generalmente son excluidos y por eso justamente, todos los países, todos los gobiernos deben asumirlos como grupos de atención prioritaria. Y, en ese sentido, entonces sí estamos hablando de personas que están en situación de discapacidad, pero también estamos hablando de otros grupos que por lo general han sido vulnerados en sus derechos y, por lo tanto, deben prioritariamente tener atención. Dentro de esa atención general que debe brindar el Estado, deben tener el derecho a ser incluidos en la educación.

\section{S.A.: ¿Podrías nombrar algunos de ellos?}

Harold Munster: Afrodescendientes, mujeres, personas que estén en situación de movilidad humana, aquellas personas que han sido abusadas sexualmente, todos aquellos grupos que independientemente de su condición hayan estado excluidos, hayan estado vulnerados de alguna manera. Garantizar los derechos de los grupos de atención prioritaria implica asumir que somos únicos, irrepetibles en capacidades, étnicamente; asumiendo nuestro género, asumiendo posiciones políticas, religiosas, condiciones biológicas, etc. En esa amalgama, en esa diversidad, es donde justamente la educación debe abordar estrategias para garantizar derechos, pero de una manera efectiva, es decir, que independientemente de su condición o de su situación todas y todos puedan tener acceso a la educación y, en segundo lugar, que ese acceso sea efectivo, que contribuya al desarrollo de la personalidad, que sea integral y multifacético, que prepare a la persona para la vida.

S.A.: Esto que mencionas sobre garantizar derechos...¿qué implicaciones tiene el enfoque de derechos ante quizás otro tipo de enfoques que pueda tener la educación inclusiva? 
Harold Munster: Ha habido muchos enfoques para hacer inclusión educativa, no educación inclusiva; pero, por lo general, han sido posiciones extremas; por lo tanto, ha habido enfoques muy clínicos, ha habido enfoques muy sociales, desde el extremo de la observación de lo social, y eso ha traído como consecuencia que nos hayamos polarizado en la búsqueda de estrategias. Por ello, muchos nos centramos ahora en un enfoque de garantía de derechos, porque garantizar derechos implica asumir a los seres humanos en su biopsicosocialidad, ver lo social, pero que en esa concepción de lo social esté implícito lo histórico, los aspectos generales que son compartidos y los individuales; implica ver lo cultural del ser humano, que lo ha formado y que, por lo tanto, lo mediatiza, y ver también lo espiritual, más allá de cómo haya adquirido su espiritualidad, asumirla como el escaño más alto del desarrollo de la personalidad. Por lo tanto, un enfoque de derechos conlleva una multifactorialidad, supone asumir muchos aspectos: biológicos, psicológicos, sociales, históricos, culturales, espirituales, de tal manera que esa concepción de diversidad del ser humano vista desde la educación permita formular estrategias que logren el desarrollo integral y multifacético que se necesita.

Es súper importante asumir una integralidad, que el enfoque bajo el cual se establezcan estrategias de inclusión, sea lo más integral posible, que sea lo más diverso posible, que sea lo más interdisciplinar posible, que sea lo más interfactorial posible, de tal manera que quepa la concepción de diversidad. Que el ser humano pueda ser observado en todas sus multifunciones, pueda ser asumido desde su multi-participación social. Entonces todo enfoque o todo paradigma educativo que se asuma desde un extremo no será bueno para el tema de la inclusión, siempre quedarán aspectos no observados y por supuesto no concebirá una estrategia educativa para hacer inclusión educativa.

Si me preguntas cuáles son los mejores enfoques, diría que hoy hay una tendencia muy fuerte, sobre todo en Latinoamérica, a asumir o el enfoque biopsicosocial, porque comprende tres dimensiones importantes, que aglutinan a su vez otros factores. Pero también hay una fuerte tendencia desde las ciencias sociales a asumir un enfoque social, donde en lo social se incluye lo natural del ser humano, es decir, lo biológico, se incluye ese reflejo subjetivo de la realidad objetiva y también incluye 
todo lo histórico y todo lo cultural y espiritual de las personas. Para mí el mejor enfoque es aquel que más aspectos abarque a la hora de asumir la diversidad humana y aquel que permita establecer claras estrategias para llevar a cabo acciones precisas de inclusión educativa.

\section{Beatriz García: ¿Cuál es tu opinión en cuanto al término discapaci- dad? ¿Hablar de discapacidad no es en sí mismo un lenguaje que puede generar exclusión o discriminación? En este sentido, ¿cómo se mira a las personas que tienen necesidades educativas especiales: como personas con discapacidades o como personas con potencia- lidades?}

Harold Munster: Bueno, el tema de las discapacidades es muy polémico, esta polémica está marcada por muchos vacíos de ciencia, por muchas contradicciones de ciencia y, sobre todo, por muchas imprecisiones de ciencia. La comunidad de personas con discapacidad, usando la Convención sobre los derechos de las personas con discapacidad, apoyados en organizaciones como la Organización Mundial de la Salud, han venido históricamente tratando de defender mucho un lenguaje positivo y, sobre todo, una concepción que no vulnere sus derechos, más bien, que les permita garantizar sus derechos.

En este sentido, se logró en la Convención sobre los derechos de las personas con discapacidad, por la Organización de Naciones Unidas, asumir a estas personas como seres humanos que tienen discapacidad, llamarlas como personas con discapacidad. Se lo asumió así, hace muchos años, cuando realmente la discapacidad ni siquiera era observada por muchos países. Fue una ganancia histórica en su momento, pero ya es hora de trascenderla. En ese momento ni siquiera la discapacidad era una condición humana, y ese fue otro logro de la Convención, visibilizar y sobre todo garantizar que los países que la firmaron puedan asumir la discapacidad como una condición humana. Ahora, si bien es cierto que es una condición humana, por lo menos yo como profesional, estoy en contra de asumirla como una condición de vida, si yo asumo la discapacidad como condición de vida, y así es el discurso de la mayoría de gobiernos, sobre todo en América Latina y también en Europa, asumo hablar del "con", lo que implica que la persona tiene que vivir con eso. 
Persona con discapacidad es persona que tiene algo, que es concebido como algo individual, sin embargo, eso ni siquiera es así, la discapacidad no es algo individual, la discapacidad no la tienen las personas, y entonces allí, bajo esa concepción inadecuada, es donde se pretende concebir estrategias educativas, que por supuesto bajo esta concepción, bajo estos criterios de ciencia, no resultan en inclusión, no resultan estrategias incluyentes o inclusivas.

\section{S.A.: Y quizás afecta a la propia psicología de las personas, porque, si tú le hablas a un niño o niña diciéndole que es una persona disca- pacitada, derrumbas incluso la aceptación de sí mismo.}

Harold Munster: Ya la Convención acordó que no debía llamarse discapacitados o discapacitadas, sino personas con discapacidad, que obviamente pudiera entenderse que es un logro; a mi modo de ver personal y sobre todo profesional, si fue un logro histórico en su momento, hoy por hoy sigue vulnerando derechos, porque la discapacidad hay que asumirla no como lo que individualmente sufre una persona y con lo que está condicionada de por vida; lo que es condición es la condición biológica, lo que es condición es entonces lo psicológico, lo que es condición es aquello que podría ser asumido desde el punto de vista médico, desde el punto de vista de salud y aun así es relativo. Una persona que tiene discapacidad visual y la condición de no poder ver, obviamente es una condición que puede ser para toda la vida, así también hay condiciones de salud que afectan aspectos físicos, lo psicológico, lo intelectual, lo auditivo y, a lo mejor, aquellas personas puedan vivir con esa condición biológica, psicológica, médica, de salud, para toda la vida, pero eso no es discapacidad. Y ahí está el conflicto tan grande y los vacíos de ciencia que han existido.

Discapacidad entonces, es un concepto mucho más amplio y que se sale de la condición psicológica y biológica que tiene el ser humano, ahí se incluye un grupo de aspectos que son más bien factores. Entonces saliéndonos de estas concepciones médicas que condicionan al ser humano, habría que definir la discapacidad como una situación social del desarrollo humano, esto es muy beneficioso para la conceptualización de la discapacidad, pero, sobre todo, para asumir estrategias realmente 
inclusivas. Asumir la discapacidad en lugar de como una condición de persona con, asumirla como condición social del desarrollo humano de personas en situación de discapacidad, que es una concepción que va mucho más allá de cuestiones lingüistas o gramaticales, pues habla de la concepción de ser humano.

Si nosotros asumimos la discapacidad desde la condición social del desarrollo humano, estamos diciendo que la discapacidad puede ser superable. Si yo digo que es situacional, entonces estoy induciendo y dando las condiciones para generar estrategias a través de las cuales pueda superarse la condición de discapacidad, por eso es una situación social del desarrollo humano. Y muchos dirían, bueno, pero puede ser que siga siendo sordo toda la vida, o puede ser que siga sin ver toda la vida o que no pueda movilizarse de por vida. Y sí, es verdad, esa es la condición biológica, esa es la condición psicológica, esa es la condición intelectual; pero, sin embargo, cuando la sociedad logre equiparar las capacidades que tiene una persona, cualquiera que sea en su diversidad, y las oportunidades de desarrollo y autonomía funcional para que aquella persona logre alcanzar un desarrollo integral y multifacético de su personalidad, entonces se superará la situación de discapacidad.

Así pues, podemos ver a una persona como Xavier Torres, siempre pongo el ejemplo de Xavier Torres o del presidente Lenin Moreno; Xavier Torres desde el Consejo Nacional para la lgualdad de Discapacidades (CONADIS), tiene acceso a autos adaptables y a toda la tecnología y la ayuda técnica para poderse movilizar, para actuar y para ejercer la presidencia del CONADIS. Así, estando en silla de ruedas logra superar su situación de discapacidad. Pero, si Xavier Torres tiene que subirse en el trole o coger un bus, entonces regresa a su situación de discapacidad. S.A.: Entonces...i qué supone eso para el Estado ayudar a que las personas superen esa situación y logren su desarrollo integral, porque no es solamente un asunto de voluntad personal, sino que también implica unas condiciones. ¿Cuáles son esas condiciones que el Estado debería garantizar, que no son solamente educativas, para lograr su desarrollo? Harold Munster: Equiparar oportunidades. Y dicho así parce fácil, pero no lo es. Lo primero que el Estado debe considerar, de manera general, es garantizar todas las ayudas técnicas que posibiliten la funcionalidad. Y cuando digo ayudas técnicas, me refiero a ayudas técnicas para el 
hogar, para la sociedad en general, para la escuela o para los centros de trabajo; es decir, que la persona pueda tener acceso. Estoy hablando de tecnologías de la información y la comunicación, estoy hablando de tecnologías generales de adaptabilidad, estoy hablando de acceso a todas las infraestructuras, a todo lo que el Estado pueda garantizar para equiparar oportunidades.

Estas personas tienen determinadas discapacidades auditivas, visuales, intelectuales, físicas, de lenguaje y la comunicación, pero ante esas discapacidades, se presentan estas oportunidades de desarrollo que les permiten ganar en autonomía funcional y que, a partir de esa ganancia en autonomía funcional, pueden incrementar su participación social, porque somos seres sociales y, por lo tanto, solo en sociedad, solo en actividad social, las personas nos podemos desarrollar integral y multifacéticamente.

Nosotros hacemos pareja socialmente, hacemos familia socialmente, estudiamos socialmente, trabajamos socialmente, nos procreamos socialmente, aunque tengamos momentos de intimidad, pero al final es un acto social. Para poder alcanzar ese desarrollo integral y multifacético, obviamente tenemos que tener autonomía funcional y para tener autonomía funcional tenemos que tener garantizado por parte de toda la sociedad, dígase Estado, dígase la sociedad civil, dígase la familia, dígase las escuelas, dígase todas las instituciones y ambientes sociales, las mismas oportunidades.

\section{S.A.: ¿Tú crees que el Estado ecuatoriano ha tenido algunos avances en relación a eso que estás planteando o hay mucho por hacer en ese sentido?}

Harold Munster: Yo como no soy triunfalista, soy de los que se centran más en lo que falta y no en lo que tenemos. Para ser justo, creo que el Estado ecuatoriano ha avanzado mucho, pero creo que le falta mucho más de lo que ha avanzado, le falta mucho más por lograr. En este sentido, mi percepción como profesional es que se trabaja por campañas, es que se trabaja por misiones, pero no hay un sistema. La atención integral de las personas con discapacidad tiene que ser un sistema, un 
sistema que sí está bien legislado en el Ecuador, pero está ahí en la ley, y a la hora de concretar esa ley, entonces nos topamos con campañas, con acciones aisladas, acciones fuera de un sistema que, pese a estar en la ley, no se logra aplicar en la vida práctica. Y entonces no hacemos nada con leyes y reglamentos muy bien concebidos, cuando a la hora de hacer inclusión social, a la hora de hacer inclusión educativa, a la hora de garantizar los derechos y de equiparar esas oportunidades, nos encontramos con situaciones que más allá de no ser incluyentes, son excluyentes para los grupos de atención prioritaria.

\section{S.A.: ¿Cómo ves la situación en el sistema educativo con respecto a garantizar esos derechos de personas con discapacidades, lo esta- mos haciendo, no lo estamos haciendo, en qué medida hay avances o no los hay? ¿cómo ves los centros educativos en Ecuador en relación a este tema de garantizar ese desarrollo de niñas, niños y jóvenes ensituación de discapacidad?}

Harold Munster: En el tema de la educación, que por principio debe contemplar una inclusión educativa, mi gran preocupación y ocupación está en que las acciones que se toman o, al menos las que se evidencian aún siguen siendo aisladas y no asumen un sistema de atención integral. Yo haría mención a tres o cuatro cuestiones que deben incluirse como sistema, lo que no se ha logrado aún. No podemos hacer inclusión educativa sin una reconceptualización de la discapacidad en el Ecuador, que incluya la propia definición de la persona con discapacidad y la clasificación de discapacidades en el país. Eso hoy está muy mal, no se logra contextualizar, no se logra resolver, incluso hay unas observaciones de Naciones Unidas a la presentación del informe de discapacidades que hizo Ecuador, a las que tenía que dar respuesta justamente antes de diciembre de este año. La primera observación que le hacen a Ecuador es que tienen una conceptualización que vulnera derechos; la definición de persona con discapacidad que tienen Ecuador está centrada en las deficiencias, y esto no es un tema solamente de definición, sino que las definiciones se convierten en herramientas de trabajo. Las personas se paran sobre una definición y con esto crean estrategias, esto es lo que define qué tipo de acciones se van a hacer, en este caso de inclusión educativa. 
Entonces con una definición de discapacidad que vulnera derechos, que está centrada en lo individual y que está centrada en la deficiencia, no se logra encontrar un soporte metodológico para hacer inclusión educativa. Si además de eso encontramos una clasificación de discapacidades que está totalmente trastocada, donde es posible encontrar un autismo en una discapacidad intelectual, donde no existe la discapacidad de lenguaje, sino que las condiciones de salud que afectan el lenguaje están también asociadas a la discapacidad intelectual, donde no está definida una discapacidad psicosocial, sino discapacidades mentales y psicológicas. Todo esto confunde y no permite definir estrategias para la atención integral de las personas con discapacidad.

La otra situación que se debe resolver la mencionábamos al inicio, darnos cuenta de que no hacemos educación inclusiva, los conceptos que manejan quienes hacen políticas públicas de educación, que pretenden hacer inclusión educativa y permiten crear estrategias y acciones, son muy generales. Por lo tanto, el Ministerio de Educación y la sociedad civil que trabaja en educación deben replantearse esta conceptualización y partir de que lo que debemos hacer es una inclusión educativa, lo que implica llevar a cabo estrategias mucho más precisas, más concretas. No es lo mismo decir, voy a hacer educación inclusiva, que plantearse lo que debo hacer para garantizar los derechos de esas personas a través de acciones concretas que me permitan acercarme a ese principio.

\section{S.A.: Esas acciones concretas tienen que ver con el tipo de discapa- cidad?}

Harold Munster: Claro, esto es simple, si usted indaga ahora en un buscador sobre educación inclusiva, va a ver conceptos muy generales y herramientas muy generales; si usted busca inclusión educativa, va a encontrar acciones mucho más concretas, conceptualizaciones, clasificaciones que le van a permitir aterrizar a la acción que garantice la inclusión como condición o como principio de educación. Por ello, este sería un aspecto esencial que debe considerarse para hacer una educación realmente inclusiva.

El otro aspecto de gran importancia es la combinación entre la educación general y la educación especializada, ese es un gran problema que 
todavía no se resuelve en Ecuador, y no solamente en Ecuador, no se resuelve en muchos países, sobre todo en Latinoamérica.

\section{S.A.: ¿Qué significa eso?}

Harold Munster: Es preguntarse qué papel debe jugar la educación especializada en el tema de la inclusión, es preguntarse si habrá inclusión en la educación especializada, ¿en la educación especializada también se habla de inclusión? El Ministerio de Educación tiene una subsecretaría que habla de educación especializada y educación inclusiva; nótese cómo las propias conceptualizaciones, de acuerdo a cómo se asumen desde la ciencia, definen estructuras gubernamentales. Cuando en realidad debería haber acciones sobre la inclusión educativa tanto para la educación especializada, como para la educación general, eso qué quiere decir entonces, que tanto la una como la otra deben asumirse como inclusión educativa, donde las acciones que se están haciendo sobre los grupos de atención prioritaria con los que se trabaja deben ser inclusivas, esto en un primer momento.

En un segundo momento, es también súper importante el papel relacional que debe haber entre la educación especializada y la educación regular, haciéndose en ambas educación inclusiva. En este sentido hay un divorcio, hay una lejanía conceptual, pero también práctica de la educación especializada y de la educación regular para hacer inclusión educativa, cuando la idea debe ser que estén muy relacionadas. La educación especializada debería ser asesora, debería ser orientadora, porque está mucho más empapada de las temáticas de garantía de derechos y temas de inclusión, tiene muchos más recursos, tiene más preparación, o se supone que debe tener más preparación en temas relacionados a tecnología en esta área, para poder asesorar, orientar y acompañar las acciones de inclusión educativa de la educación regular.

S.A.: ... la idea es que una persona que tiene discapacidad, no necesariamente tiene que estar en un centro de educación especializada, es decir, puede entrar en educación regular si hay condiciones a nivel de infraestructura y formación docente para que eso pueda darse. 
Harold Munster: La utopía debe ser, o es que la educación especializada sea una educación de tránsito, ya sea en una educación aparte, independiente a la educación regular o dentro de la propia educación regular y Fe y Alegría ha venido dando pasos en ese sentido. El modelo de inclusión educativa que tiene Fe y Alegría implica eso, implica aulas especializadas de educación inicial, ya sea de Educación Inicial Uno, o de Educación Inicial Dos. Eso es incluir la educación especializada en la educación regular. Es concebir una estrategia en la cual haya una imbricación entre la educación especializada y la educación regular, pero una educación especializada que prepara para el tránsito, transitoria, donde desde la propia institución regular, se vaya preparando a los niños y niñas que tengan algún tipo de situación de discapacidad o a otro grupo de atención prioritaria, para que sea incluido en las aulas de educación regular. La educación especializada se debe concebir de esa manera, en una estrecha relación con la educación regular, las dos hacen inclusión educativa y una por ser especializada, repito, debe estar ahí en función de orientar, asesorar, acompañar las acciones que se hagan desde la educación regular.

S.A.: ¿Cómo ves tú el que los centros de educación especializada a su vez se especialicen en determinados tipos de discapacidad, porque, quizás algunos centros consideren que hay una gran diversidad de discapacidades, que no pueden atender todas, dado que no cuentan con la formación y los docentes no pueden abarcar tanto cuando en un mismo salón de clases hay estudiantes con variedad de discapacidades. Ese tipo de problemas se pueden estar presentando en centros educativos y la tendencia es a concentrarse solamente en, por ejemplo, niñas y niños sordos. ¿Cómo ves tú esa situación, crees que debería tenderse hacia allá o debería tenderse a una atención de las diversidades de discapacidades que existen?

Harold Munster: Como te decía al principio, todos los extremos son malos en los centros educativos. Si nosotros concebimos una educación especializada entre muros y si es más especializada mejor, escuela para sordos, escuela para discapacidad visual, el otro día me encontré con una escuela para discapacidad física, cuando con esas personas es prácticamente un tema de accesibilidad, de movilidad, no es un tema 
intelectual, no es un tema senso-perceptivo. Y bueno, mientras más específica sea la educación especializada, mucho más excluyente va a ser, porque va a sacar a las niñas, a los niños, a las personas de su ambiente natural, ¿cuál es su ambiente natural? bueno, el ambiente de todos los niños y niñas, en su diversidad de capacidades, ese es el ambiente natural, y es el ambiente propicio para poder lograr autonomía funcional y participación social. Por lo tanto, cuanto más especializada sea, refiriéndome a atender a un solo tipo de discapacidad, desde mi punto de vista como profesional, tiende a la exclusión, no a la inclusión.

\section{S.A.: ¿Cuál es el otro extremo?}

Harold Munster: El otro extremo de la mal llamada educación inclusiva o de unas inadecuadas acciones de inclusión educativa está en, sin ningún tipo de estrategia sistémica, poner niños, digo poner con ese énfasis, no es incluir, poner niños con cualquier tipo de discapacidad en aulas de educación regular, eso no es inclusión, eso es atentar contra una real inclusión, más bien, eso genera exclusión. Porque entonces, maestros, maestras, que no tienen estrategias, fijate que ni siquiera es un tema de conocimientos, puede ser muy conocedora, pero, como no hay una estrategia sistémica, que vaya mucho más allá de la preparación del docente, que tiene que ver con higiene, ambiente escolar, organización escolar, ayudas técnicas, con todo un grupo de condiciones que garantizan inclusión, no se logra una verdadera inclusión, lo que se logra es tener niños y niñas a veces sin siquiera realizar actividades en las aulas, entonces eso genera exclusión. Eso genera frustraciones y es el otro extremo negativo.

Ahora me dirás ¿cuál es el punto medio? el punto medio es lograr lo que decía, lograr una interrelación entre acciones de educación especializada y acciones de educación regular, siendo éstas, acciones de inclusión educativa. Una educación especializada y una educación regular integradas en acciones sistémicas de inclusión educativa, ese sería el concepto mediador entre los dos extremos que decía.

Eso es muy difícil de lograr cuando tienes un centro de educación especializada por un lado para personas sordas, un centro para personas 
con discapacidad intelectual $y$, entonces tienes en ese entorno varios centros de educación regular, eso es muy difícil de lograr. ¿Cuándo podremos lograr inclusión educativa? cuando nos demos cuenta de que la inclusión educativa debe ser preparada, hay que concebirla como proceso y entonces desde ese proceso lograr acciones de educación especializada en los centros de educación regular, como viene haciendo muy bien Fe y Alegría. Es concebir aulas de educación especializada en las primeras edades, de tal manera que se pueda ir logrando una preparación. Y entonces dirán, eso es meter educación especializada en la educación regular y eso puede generar exclusión, no, porque lo único que hago diferente a la educación regular es el trabajo que hago en aula, pero independientemente del trabajo en aula, las niñas y niños en situación de discapacidad participan en los matutinos, en los vespertinos, en las actividades de las que forman parte el resto de niñas y niños que no tienen esa situación de discapacidad o que no están en situación de discapacidad. Entonces, de manera natural las niñas y los niños están incluidos con sus pares, están en su ambiente natural, solamente se están haciendo acciones con ellos de manera independiente en las aulas, garantizando un tránsito a la educación regular, de una manera previamente concebida. Con esto entonces lograríamos que una maestra de primero o de segundo grado de básica asista a las aulas de educación especializada, para irse nutriendo de todo lo que se está haciendo con esos niños, de cómo van avanzando, de cómo son las estrategias pedagógicas que se están desarrollando y así ella también se va preparando para ese tránsito, no solamente los niños y niñas.

\section{S.A.: Eso supone también de los maestros y maestras una sensibili- dad en torno a la atención a niños, niñas y adolescentes con disca- pacidad. Porque podemos encontrar maestras y maestros que no quieren tener en sus aulas niños o niñas con discapacidad porque sienten que les implica más trabajo o que les supera. ¿Cómo ves el tema de actitud?}

Harold Munster: Los seres humanos pensamos y actuamos de acuerdo a lo que vivimos, esto quiere decir que mis actitudes están determinadas por mis vivencias, por mi preparación, por lo tanto, hay que vivir la discapacidad. Si usted es maestra o maestro y llega una persona en 
situación de discapacidad, lo primero que usted hace es asustarse, no importa su nivel de preparación, porque usted leyó, buscó, se preparó en la universidad, hizo postgrados y maestrías, pero la realidad es mucho más rica que lo que uno encuentra en los libros. Entonces para esa, yo diría, modificación de actitudes, ese poder trabajar asumiendo la discapacidad del otro o de la otra, se precisa de la vivencia, no está ni siqueira en la sensibilización, que es algo que se mueve por los afectos, yo diría que está en la concienciación. De tal manera, que estos niños y niñas, como diríamos, no te caen de sorpresa, sino que ya los vienes observando en las variantes de educación especializada que se hagan desde la educación regular, cuando tú estás participando de los juegos de tus estudiantes con ellos incluidos, cuando los ves actuar en una presentación, entonces, ya no te resulta raro, ya no te resulta difícil trabajar con ellos, porque ya estás sensibilizado al inicio y concienciado luego, esto como primer elemento.

Como segundo elemento es donde retomo la preparación, pero no la preparación académica, de cursos, a la que no le estoy quitando méritos, pero no es suficiente, yo me refiero a la formación continua y constante de los maestros y las maestras en función de poder atender efectivamente la discapacidad o cualquier otra situación que precise utilizar procedimientos específicos para las niñas, niños y adolescentes. Por lo tanto, la preparación docente, la formación constante y sistemática hacia estos objetivos es uno de los aspectos que constituyen una necesidad, un requerimiento para poder hacer inclusión educativa que sea efectiva.

Otra cosa importante es poder contar con tecnologías, sobre todo con las tecnologías de la información y las comunicaciones, que va mucho más allá de tener internet, que van mucho más allá de darles computación a las y los muchachos, que van más allá de hacer informática. Es necesario contar con recursos que te permitan poder incorporar determinadas metodologías, determinadas didácticas de trabajo, que les ayuden a incrementar su autonomía funcional para así apropiarse del contenido de la enseñanza, los niños y las niñas, las personas que están en situación de discapacidad tienen el derecho de apropiarse del contenido de la enseñanza. Pero esa apropiación es distinta que la de los demás; más allá de lo que yo enseñe, estoy centrado en lo que tú apren- 
des y para que ese aprendizaje sea significativo tiene que estar basado en la apropiación del contenido de la enseñanza. Y, para yo apropiarme del contenido de la enseñanza, en una situación de discapacidad, necesito un medio técnico, necesito tecnología, que me permita asimilar ese aprendizaje.

Otro aspecto a considerar es que las maestras y los maestros les dan un énfasis extraordinario a los ajustes curriculares, he notado que los ven como la única solución a la problemática. Para ellos y ellas si no llevas a cabo los ajustes curriculares, no haces inclusión; con esto no estoy diciendo que no hagan los ajustes, sino que hay que pensar en diversidad, pero también hay que pensar en igualdad, con esto me refiero a que la misma tecnología y el mismo recurso didáctico que le permite a un niño, niña o adolescente que esté en situación de discapacidad apropiarse del contenido de la enseñanza, puede ser apropiado para la enseñanza de los demás.

\section{S.A.: Es decir que no necesariamente un docente tiene que hacer adaptaciones curriculares para poder trabajar con niñas y niños que estén en situación de discapacidad.}

Harold Munster: Yo diría que no es un tema de ajuste, es un tema de concepción del currículo, de concepción didáctica del currículo. Por ejemplo, si un niño o niña que está en situación de discapacidad intelectual, si le voy a enseñar los fonemas y logra aprender mucho mejor con un método fónico-analítico-sintético, por qué debería aplicar este método exclusivamente a él o ella y otro distinto a los demás. Eso hace difícil la clase, hace difícil el hacer pedagógico; entonces la reflexión que debe hacerse es, que si con un niño o niña con situación de discapacidad funciona bien un método, puedo aplicarlo a su vez a los demás, y con eso no estoy desmereciendo o minimizando el aprendizaje de los demás, porque finalmente el objetivo es que todos aprendan y aprender es apropiarse del conocimiento. Si yo logro con un mismo procedimiento y un mismo método que todos y todas, desde la diversidad de mi aula, puedan apropiarse de ese conocimiento, entonces no es necesario el ajuste. 


\section{S.A.: ¿Cuál es el tercer aspecto importante para hacer inclusión?}

Harold Munster: Sí, otro aspecto importante, para lograr hacer inclusión educativa es desacomodarme, cuando digo desacomodarme, me refiero a salirme de los esquemas tradicionales de la educación, en los cuales yo como docente esté sentado en el aula esperando que las madres y los padres de los niños y niñas que están en situación de discapacidad vengan a matricularlos en la escuela. Yo me tengo que desacomodar y contribuir a hacer una detección temprana y una inclusión educativa lo más pronto posible. Los estudios demuestran que las familias de niñas y niños que están es situación de discapacidad están también en tensión, porque eso es situacional, entonces la tendencia es a ocultarlo, es a sentir vergüenza, la tendencia es a no socializarlo, a veces por un desconocimiento o por una inadecuada percepción social de la problemática que enfrenta ese niño o niña. En este sentido, las escuelas tienen que desacomodarse y desde la concepción, como la que tiene Fe y Alegría, de comunidad educativa, irse al barrio, y esto no lo debe hacer solo; en esto Fe y Alegría tiene mucha experiencia, es necesario aliarse con todas las entidades comunitarias, aliarse a los centros de salud de primer nivel de prevención, aliarse a todo aquel que permita, facilite o contribuya a hacer una inclusión temprana y un acercamiento de estos niños y niñas a la escuela y entonces hacer inclusión educativa.

\section{S.A.: Y fijate que tú estás hablando de unas prácticas vistas desde el lado de la inclusión educativa, pero que realmente debería hacerse para muchas otras cosas también, ese salir a la comunidad aplica al tema de la inclusión educativa, pero también para todo lo que la educación realmente es o debería ser.}

Harold Munster: Bueno, desde ese punto de vista asumo a la escuela como el líder educativo de la comunidad, es lo que debe ser, el líder educativo, el líder formativo de la comunidad, desde mi percepción no deben ser otras entidades. ¿Quién tiene el cargo social de la formación? la familia tiene una función educativa intrínseca, que esa no se la quita nadie, esa la asumen desde la hora que lo decidieron o desde la hora en la que se convirtieron en padres por casualidad; pero la escuela no, los centros educativos tienen ese encargo social, lo tienen por ley. Por lo 
tanto, desde esa concepción de comunidad educativa tienen el encargo, tienen la preparación, tienen la metodología, de manera que solo tienen que hacerlo. Y fíjate cómo son los discursos tuyos y míos, "tienen que salir a la comunidad", y no, no hay que salir a la comunidad, es que somos parte de la comunidad. Y así lo asumimos porque así nos lo han enseñado, que la escuela tiene que salir a la comunidad, que tiene que abrirse a la comunidad, no, cuando nosotros nos demos cuenta de que somos parte y la parte más importante de la comunidad desde el punto de vista formativo, entonces, no hay que salir a hacer nada, es sentirse parte de la comunidad, y en eso Fe y Alegría tiene muchos resultados refiriéndome al tema de inclusión educativa, sobre todo en Santo Domingo, porque han salido los maestros y las maestras a hacer visitas domiciliarias, a sensibilizar a las familias a conversar con el barrio, a solicitarles que apoyen a esas familias para la matrícula de los niños y niñas. En ese sentido, existe una ganancia extraordinaria, ya que, según los múltiples aportes de la psicología infantil, sobre todo los de Vigotsky, él decía que la niñez es la etapa más sensitiva, es la etapa en la cual puedes lograr muchísimas cosas que después como adolescente o adulto ya es mucho más difícil.

\section{S.A: Así es, por eso es que ahora el énfasis está en la educación inicial, porque ya nos hemos dado cuenta de que son años funda- mentales, antes quizás no había esa claridad. Un último tema que me gustaría tratar es el tema de familia. ¿Cómo deberíamos abordar la educación y el acompañamiento de la familia en los casos de niños y niñas con discapacidad?¿Cómo debe ser el trabajo con las familias desde el punto de vista de los centros educativos?}

Harold Munster: Para yo hablar de familia tengo que empezar de arriba, yo creo que el gran problema, es el hecho de que no funcionamos en sistema, entonces hacemos parcelas, y al hacer parcelas hay un grupo de competencias gubernamentales que les toca salud, hay un grupo de competencias que les toca educación, y en esto hay que considerar que el ser humano es un ser integral, es un ser integrador en sí y cuando las entidades no se integran, entonces tenemos atención parcializada, tenemos atención que no siempre responde a u sistema. 
Y en ese sistema que te estoy dibujando así desarticulado también entra la familia, como ese contexto de actuación primaria donde se conservan y se transmiten los signos más distintivos de la cultura. A partir de ese conservar y compartir la cultura que nos mediatiza como rol inherente de la familia, la escuela tiene que comprender que no puede lograr educación inclusiva ajena a la familia, como tampoco ajena al sistema de salud, como tampoco ajena al sistema de inclusión social, como tampoco ajena a otras instancias del Estado. Pero la familia juega un papel vital, importante, lo que decía, conserva la cultura, conserva los signos más distintivos.

Si nosotros logramos una familia sensibilizada, concienciada en hacer inclusión educativa o hacer inclusión desde cualquier punto de vista, entonces tenemos ganado, yo diría, el cincuenta por ciento de la batalla, por qué, porque hay acciones que se hacen desde la institución educativa, pero que necesitan de una continuidad en el hogar, hay acciones que tienen que ver con la ganancia de la autonomía funcional y que tienen que ver con la participación social, en las que no se pueden alcanzar grandes logros solamente desde la escuela, necesitamos hacerlo también con la familia.

Beatriz García: Y cómo logramos eso en centros educativos con docentes que tienen unas cargas horarias tremendas y están movilizándose de un centro hacia otro, porque eso implica una dedicación de tiempo, también unas estrategias, implica un convencimiento de parte de quienes acompañarían a las familias para el tema de inclusión, entonces ¿cómo poder lograr esto?

Harold Munster: Mira, yo digo que el tema está en redefinir las acciones de inclusión educativa que incluso están asumidas desde el propio gobierno. Y en esto Fe y Alegría puede hacer mucho, hay que seguir haciendo educación popular, que es también incidir en las políticas públicas, que es revolucionar la realidad social, porque ese es el encargo de la educación popular. Y en ese sentido, nosotros no podemos lograr hacer inclusión educativa, y desde esa inclusión educativa apuntarle a modificar las dinámicas relacionales y sistémicas de la familia con un sistema educacional como el que tenemos hoy. 
Siguiendo esa línea, tenemos hoy maestros y maestras sin el tiempo suficiente para poder hacer un real acompañamiento y una real incidencia en la familia, maestras y maestros con una carga horaria extraordinaria, cuya organización escolar y ambiente escolar tampoco es propicio para poderlo hacer. Yo he ido a centros educativos donde el psicólogo o psicóloga del Departamento de Consejería Estudiantil (DECE) tiene una hora a la semana o cuando más, dos horas a la semana para poder trabajar con familias, para hacer orientación a las familias, no tiene el tiempo suficiente para hacer visitas domiciliares.

Siendo así, cómo hacer incidencia en la familia, el error o la limitación, para no hablar de error, creo que está en asumir que las y los educadores trabajamos con niñas, niños y adolescentes, y no nos vemos como el factor social que educa y educa en comunidad y desde la comunidad, no para la comunidad. Cómo hacer educación en comunidad educativa, cuando realmente no tenemos ni siquiera la oportunidad de poder sistematizar adecuadamente las acciones con la familia, de poder orientarle, pero más que orientar, cómo poder acompañar a esa familia en el proceso de mejorar aquella dinámica que permita generar autonomía funcional y participación social de los miembros que están en situación de discapacidad al igual que la propia familia. Eso implica hacer un rediseño completo de la educación, con más tiempo para las y los maestros, con estrategias distintas. Hay que rediseñar el modelo de los DECE y de las Unidades de apoya a la Inclusión (UDAl), porque se supone que las UDAl están para eso, se supone que los DECE están para eso. Los departamentos de inclusión deben garantizar inclusión, entonces cuando tenemos un DECE, con un psicólogo o psicóloga que no tienen ni siquiera en normativa la posibilidad de poder salir y hacer trabajo social en el barrio, entonces finalmente no podemos apuntarle a la familia. Cuando, por otro lado, tenemos una UDAI muy alejada de la realidad de la escuela y todavía, a pesar de todo lo que ha mejorado, con una visión bastante clínica de la situación de discapacidad, entonces, peor todavía. El Ministerio tiene que trascender la concepción del modelo de UDAl, de manera que permita una mejor orientación y un mejor acompañamiento en la escuela y donde deje claras las estrategias que deben tener los DECE y a través de ellos, las de los maestros y maestras para tener un acercamiento a la familia, principalmente en lo que se refiere a acompañamiento. 